\title{
Taxonomic Study of the Genus Thalia (Thaliacea: Salpida: Salpidae) from Korea
}

\author{
Sunwoo Kim ${ }^{1}$, Jung-Hye Won ${ }^{2}$, Chang-Bae Kim,* \\ ${ }^{1}$ Ecocean Co., Ltd., Incheon 406-840, Korea \\ ${ }^{2}$ Seodaemun Museum of Natural History, Seoul 120-113, Korea \\ ${ }^{3}$ Department of Green Life Science, Sangmyung University, Seoul 110-743, Korea
}

\begin{abstract}
Five species in the genus Thalia of the family Salpidae are described: Thalia cicar van Soest, 1973, Thalia democratica (Forskal, 1775), Thalia orientalis Tokioka, 1937, Thalia rhomboides (Quoy and Gaimard, 1824), and Thalia sibogae van Soest, 1973. All of these species are new to the Korean fauna. A key to the Korean Thalia species is provided.
\end{abstract}

Keywords: Urochordata, Salpida, Salpidae, Thalia, Korea, new record

\section{INTRODUCTION}

The species of the family Salpidae are free-swimming and filter-feeding marine holoplanktons. The life-cycles of the species alternate between two forms, an asexually budding solitary stage and a sexually reproducing aggregate stage. Forty species in 13 genera are currently known (Chihara and Murano, 1997; Esnal and Daponte, 1999), whereas in Korea three species in three genera have been recorded (Rho, 1967; Kim et al., 2010a, 2010b). Identification of salpid species is based mainly on the arrangement of body muscles. For identification of species in the genus Thalia, the various projections of the test, the transparent outer surface of body, are also important (van Soest, 1973). In a series of taxonomic studies on planktonic tunicate, five species in the genus Thalia were newly identified from Korean waters. This paper aims to re-describe these species which are new to the Korean fauna, with a key to Korean Thalia species.

\section{MATERIALS AND METHODS}

The specimens were collected with an IKMT net of $417 \mu \mathrm{m}$ mesh. They were fixed and stored in $4 \%$ buffered formalin or $80 \%$ ethyl alcohol, and then examined with a stereoscopic microscope (Leica MZ16 with AxioCam HRc). The abbreviation $\mathrm{M}$ indicates the muscle band. The body length was measured by using objective and ocular micrometers. Solitary zooids were measured from the terminal of the oral opening to the tip of midventral projections, whereas aggregate zooids were measured from the terminal of the oral opening to the atrial opening. Materials examined in this study have been deposited in the Seodaemun Museum of Natural History.

\section{SYSTEMATIC ACCOUNTS}

\author{
Phylum Chordata Bateson, 1885 \\ Subphylum Tunicata Larmarck, 1816 \\ Class Thaliacea Nielsen, 1995 \\ Order Salpida Uljanin, 1884 \\ Family Salpidae Lahille, 1888 \\ Subfamily Salpinae Yount, 1954 \\ ${ }^{1 *}$ Genus Thalia Blumenbach, 1798
}

Diagnosis. Solitary zooid: Test cylindrical, thickened ventrally around nucleus, with two long posterior projections. Oral opening terminal, atrial opening postero-dorsal. Five body muscles, MI to MIV continuous dorsally and ventrally, MV interrupted ventrally. MI to MIII and MIV to MV contiguous or fused in mid-dorsal line. Intermediate muscle inter-

(C) This is an Open Access article distributed under the terms of the Creative Commons Attribution Non-Commercial License (http://creativecommons.org/ licenses/by-nc/3.0/) which permits unrestricted non-commercial use, distribution, and reproduction in any medium, provided the original work is properly cited.

*To whom correspondence should be addressed

Tel: 82-2-2287-5288, Fax: 82-2-2287-0070

E-mail: evodevo@smu.ac.kr 
rupted dorsally and converge to MI. Dorsal tubercle simple and small, separated from ganglion with short distance. Branchial septum slender, extending from ganglion to gut. Endostyle thin, extending from behind oral to posterior ventrally. Gut forming a compact nucleus, stolon coiled around it.

Aggregate zooid: Body oval, rounded or pointed posteriorly. Both openings dorsal. Five body muscles continuous dorsally while interrupted ventrally. MI to MIII and MIV to MV fused in mid-dorsal line. Gut forming a compact nucleus. Testis arranged in 4 lobes. Ovary and embryo underneath MIV.

\section{1*Thaila cicar van Soest, 1973 (Fig. 1A-D)}

Thalia cicar van Soest, 1973: 193; Chihara and Murano, 1997: 1365; van Soest, 1998: 238, fig. 14.3, table 14.3; Esnal and Daponte, 1999: 1432, fig. 3.16.

Materials examined. 5 solitary zooids, Korea strait, $32^{\circ} 29^{\prime}$ $\mathrm{N}, 127^{\circ} 29^{\prime} \mathrm{E}, 4$ May 2001; 1 aggregate zooid, South Sea, $35^{\circ}$ $20^{\prime} \mathrm{N}, 130^{\circ} 12^{\prime} \mathrm{E}, 15$ Oct $2001 ; 3$ solitary zooids and 1 aggregate zooid, East Sea, 37 00’N, 129 30'E, 23 Sep 2003.

Description. Solitary zooid: $4-15 \mathrm{~mm}$ long, excluding posterior projections (Fig. 1A, B). Body flabby, relatively short (Fig. 1A, B). Test thick and smooth (Fig. 1A, B). Atrial palps large, bifurcate (Fig. 1C). Lateral projections well developed and posterior projections relatively short (Fig. 1A, B). Medioventral projections almost same length, well developed (Fig. 1B). Echinate restricted to projections and surrounding oral opening (Fig. 1A-C). Body muscles thin (Fig. 1A).

Aggregate zooid (Fig. 1D): 5-10 mm long, body oval with round posterior. Attachment organs variable in number and protruding beyond test wall left posterior.

Distribution. Pacific Ocean, Atlantic Ocean, and Mediterranean Sea.

\footnotetext{
2* Thaila democratica (Forskal, 1775) (Fig. 2A-D)

Salpa democratica Forskal, 1775: 112 (cited from Thompson, 1948).
}

Salpa spinosa Otto, 1823: 273 (cited from Berrill, 1950).

Dubreuillia cirrhosa Lesson, 1830: 433 (cited from Berrill, 1950).

Salpa cabotti Desor, 1851: 75 (cited from Berrill, 1950). Salpa mucronata Apstein, 1894: 41 (cited from Berrill, 1950). Thalia democratica: Fraser, 1947: 2; Thompson, 1948: 139, Pl. 56, fig. 3, Pl. 57, figs. 1, 2, Pl. 58, figs. 1-3, Pl. 59, figs. 1-4; Yount, 1954: 323, figs. 28, 29; Gosner, 1971: 611, fig. 24.10; Zheng, 1989: 389; Madin, 1991: 112, fig. T-16; Chihara and Murano, 1997: 1365; van Soest, 1998: 238, fig. 14.3, table 14.3; Esnal and Daponte, 1999: 1432.

Salpa (Thalia) democratica Berrill, 1950: 291, fig. 105.
Materials examined. 1 aggregate zooid, East China Sea, $31^{\circ}$ $05^{\prime} \mathrm{N}, 126^{\circ} 49^{\prime} \mathrm{E}, 4$ May 2001; 7 solitary zooids and 2 aggregate zooids, Korea strait, $32^{\circ} 29^{\prime} \mathrm{N}, 127^{\circ} 29^{\prime} \mathrm{E}, 4$ May 2001; 6 solitary zooids, Korea strait, $33^{\circ} 29^{\prime} \mathrm{N}, 127^{\circ} 45^{\prime} \mathrm{E}$, 5 May 2001; 14 solitary zooids and 13 aggregate zooids, Korea strait, $33^{\circ} 00^{\prime} \mathrm{N}, 127^{\circ} 30^{\prime} \mathrm{E}, 5$ May $2001 ; 11$ solitary zooids and 58 aggregate zooids, East China Sea, $24^{\circ} 40^{\prime} \mathrm{N}, 125^{\circ} 00^{\prime} \mathrm{E}$, 7 May 2001; 1 aggragate zooid, South Sea, $34^{\circ} 05^{\prime} \mathrm{N}, 127^{\circ}$ $96^{\prime} \mathrm{E}, 4$ Oct 2007; 6 aggregate zooids, South Sea, $34^{\circ} 05^{\prime} \mathrm{N}$, $127^{\circ} 96^{\prime} \mathrm{E}, 1$ Sep 2008 .

Description. Solitary zooid: $2-8 \mathrm{~mm}$ long, excluding posterior projections (Fig. 2A). Body oval or elongated cylindrical (Fig. 2A). Test smooth (Fig. 2A). Atrial palps simple and straight, not bifurcated (Fig. 2C). Medioventral projections small and lower one longer than upper one (Fig. 2B). Lateral projections small. All projections echinate (Fig. 2A-C). Body muscles thin (Fig. 2A).

Aggregate zooid (Fig. 2D): 1-8 mm long. Body oval with bluntly projected posteriorly. Test smooth. Attachment organs variable in number and protruding short beyond test wall left posterior. Nucleus bearing a projection posterior (nucleus projection).

Distribution. Pacific Ocean, Atlantic Ocean, Indian Ocean, Mediterranean Sea, and Red Sea.

Remarks. This species was reported previously in the "List of Animals in Korea" (The Korean Society of Systematic Zoology, 1997) under Salpa mucronata without any description or remarks. In the report, the Korean name "salpa" referring to North Korean name without any source was presented. Therefore, in this study, this species is reported as a new record to the Korean fauna with a new Korean name referring to the small body size of this species.

\section{${ }^{3 *}$ Thaila orientalis Tokioka, 1937 (Fig. 3A-D)}

Thalia democratica var. orientalis Tokioka, 1937: 226, Pl. 14, fig. 1; Tokioka, 1967: 230; Yamaji, 1984: 448, Pl. 139, figs. 7a-c.

Thalia orientalis Madin, 1991: 112, fig. T-17; Chihara and Murano, 1997: 1365; van Soest, 1998: 238, fig. 14.3, table 14.3; Esnal and Daponte, 1999: 1432, fig. 3.15.

Materials examined. 1 aggregate zooid, Korea strait, $32^{\circ} 29^{\prime}$ N, $127^{\circ} 29^{\prime} \mathrm{E}, 4$ May 2001; 2 solitary zooids, Korea strait, $33^{\circ} 00^{\prime} \mathrm{N}, 127^{\circ} 30^{\prime} \mathrm{E}, 5$ May 2001; 1 solitary zooid, Korea strait, $33^{\circ} 59^{\prime} \mathrm{N}, 128^{\circ} 17^{\prime} \mathrm{E}, 6$ May 2001; 2 solitary zooids, South Korea, $35^{\circ} 06^{\prime} \mathrm{N}, 130^{\circ} 01^{\prime} \mathrm{E}, 12$ Oct $2001 ; 6$ solitary zooids and 3 aggregate zooids, East Sea, $38^{\circ} 30^{\prime} \mathrm{N}, 129^{\circ} 49^{\prime} \mathrm{E}$, 15 Oct 2001 ; 1 solitary zooid, East Sea, $36^{\circ} 30^{\prime} \mathrm{N}, 130^{\circ} 32^{\prime} \mathrm{E}$, 15 Oct 2001; 65 solitary zooids and 10 aggregate zooids,

Korean name: ${ }^{1 *}$ 둥근탈리아살파 (신칭), ${ }^{2 *}$ 작은 탈리아살파 (신칭), ${ }^{3 *}$ 긴꼬리탈리아살파 (신칭) 

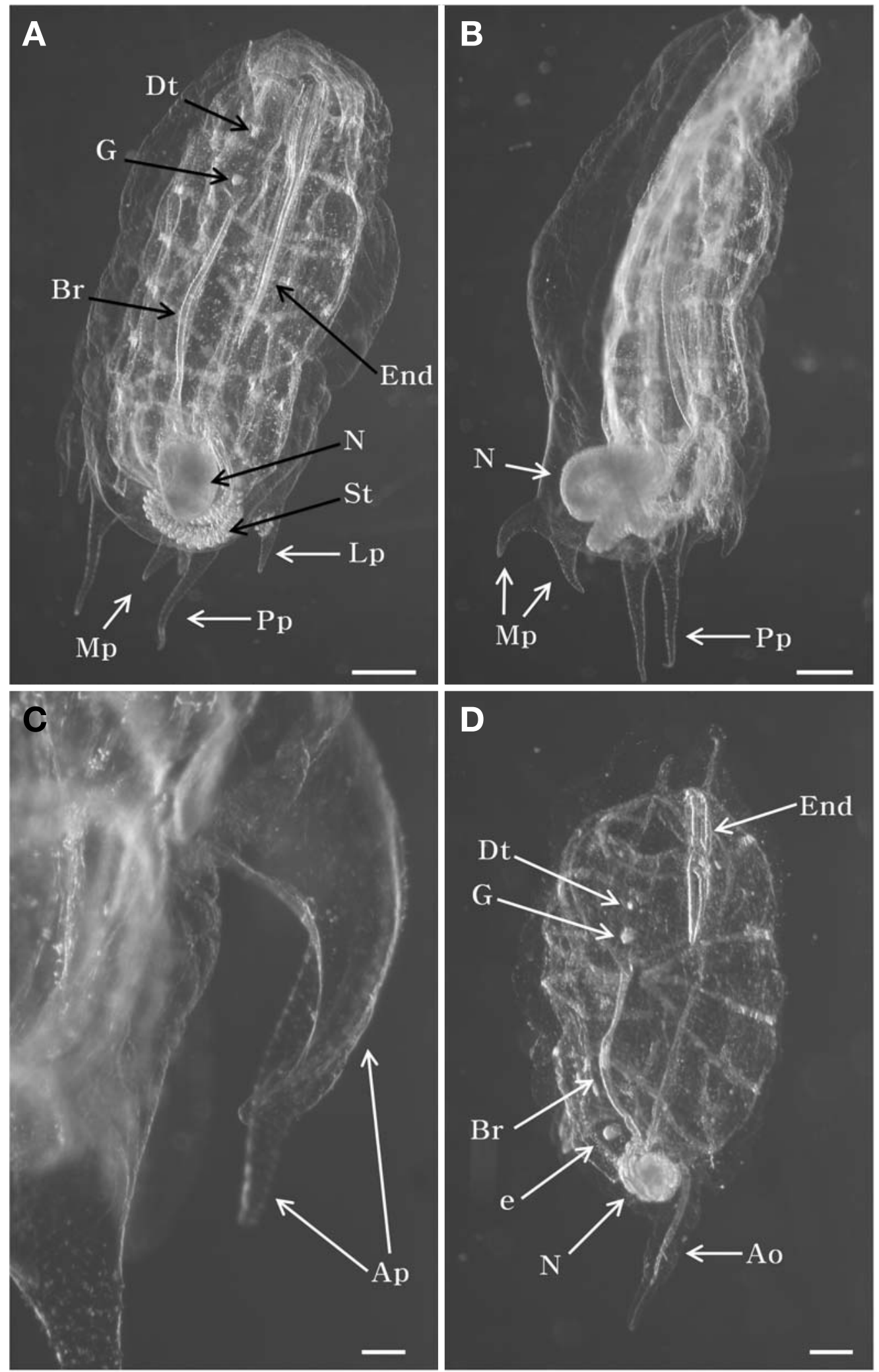

Fig. 1. Thalia cicar. A-C, Solitary zooid. A, Ventral view; B, Lateral view; C, Lateral part. $D$, Aggregate zooid, ventral view. Ao, attachment organ; $\mathrm{Ap}$, atrial projection; $\mathrm{Br}$, branchial septum; $\mathrm{Dt}$, dorsal turbercle; e, Embryo; End, endostyle; G, ganglion; Lp, lateral projection; Mp, medioventral projection; $\mathrm{N}$, nucleus; $\mathrm{Pp}$, posterior projection; St, stolon. Scale bars: $\mathrm{A}, \mathrm{B}, \mathrm{D}=1 \mathrm{~mm}, \mathrm{C}=0.1 \mathrm{~mm}$. 

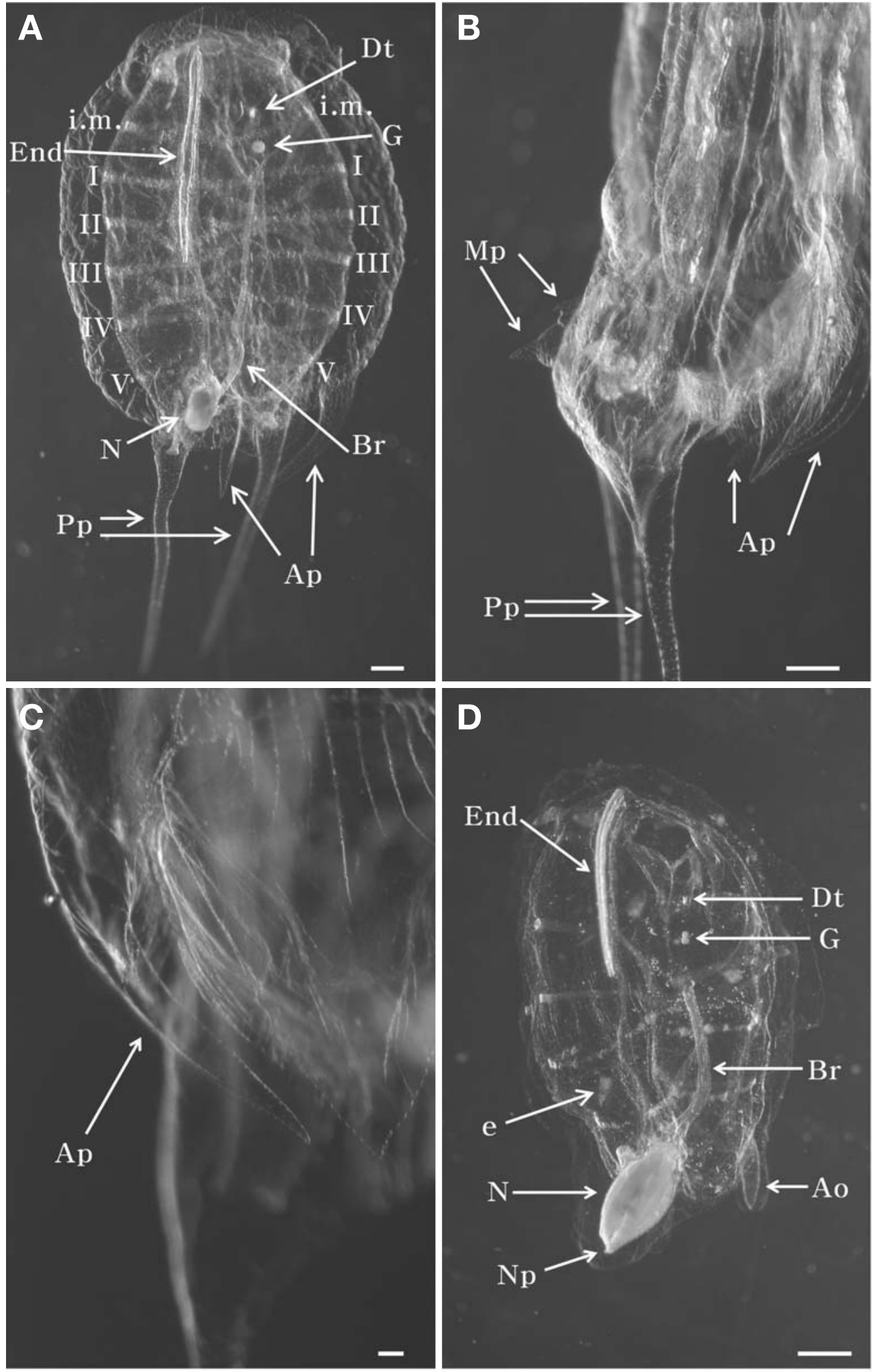

Fig. 2. Thalia democratica. A-C, Solitary zooid. A, Ventral view; B, Lateral part; $C$, Lateral part. $D$, Aggregate zooid, ventral view. Ao, attachment organ; Ap, atrial projection; Br, branchial septum; Dt, dorsal turbercle; e, Embryo; End, endostyle; G, ganglion; i.m., intermediate muscle; Mp, medioventral projection; $\mathrm{N}$, nucleus; $\mathrm{Np}$, nucleus projection; Pp, posterior projection; I- $\mathrm{V}$, body muscle. Scale bars: $A, B=0.5 \mathrm{~mm}, C=0.2 \mathrm{~mm}, D=1 \mathrm{~mm}$. 

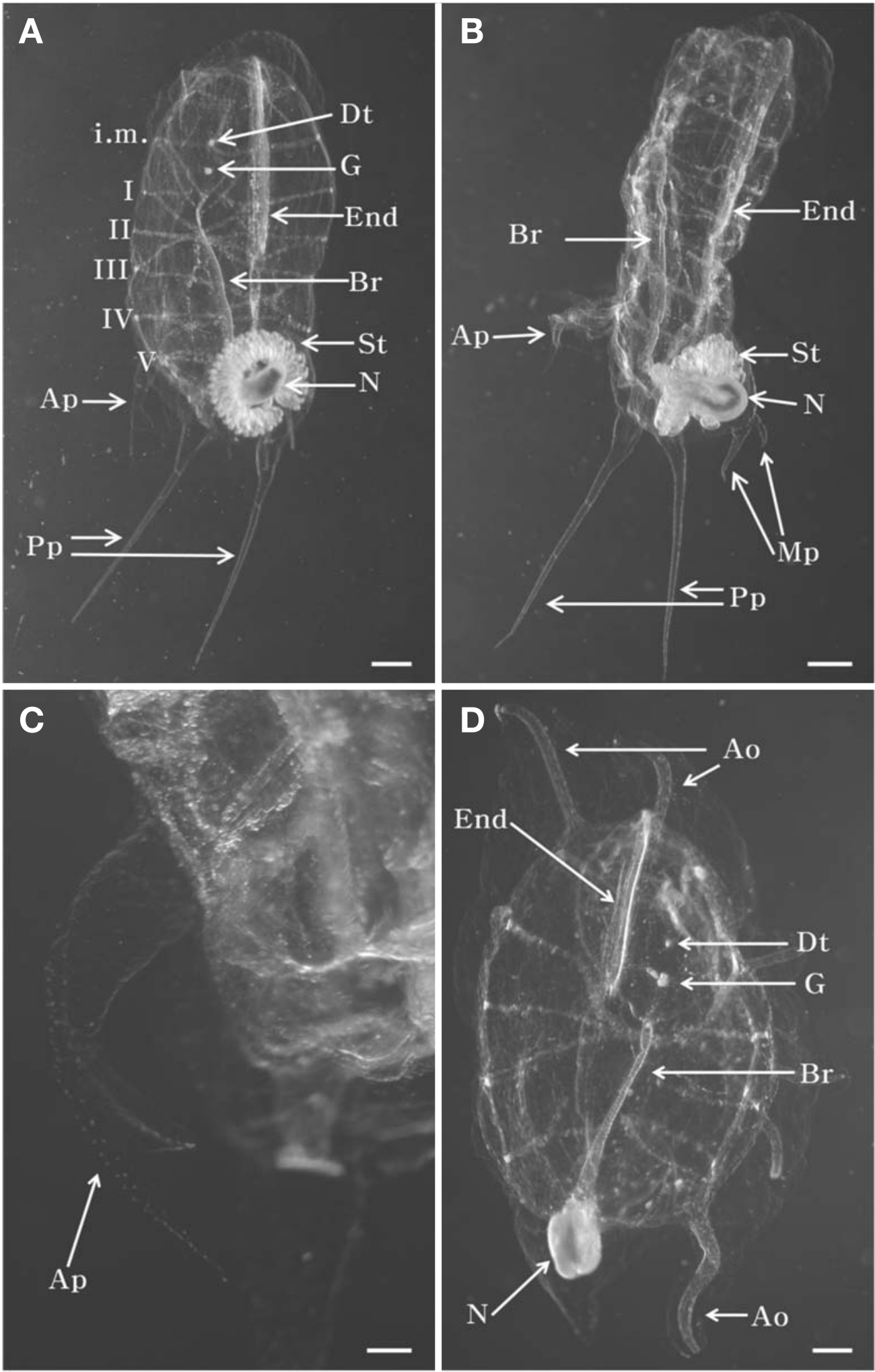

Fig. 3. Thalia orientalis. A-C, Solitary zooid. A, Ventral view; B, Lateral view; C, Lateral part. D, Aggregate zooid, ventral view. Ao, attachment organ; $\mathrm{Ap}$, atrial projection; $\mathrm{Br}$, branchial septum; $\mathrm{Dt}$, dorsal turbercle; e, Embryo; End, endostyle; G, ganglion; i.m., intermediate muscle; Mp, medioventral projection; $\mathrm{N}$, nucleus; $\mathrm{Np}$, nucleus projection; Pp, posterior projection; St, stolon; I-V, body muscle. Scale bars: $A, B, D=1 \mathrm{~mm}, C=0.2 \mathrm{~mm}$. 
South Korea, $35^{\circ} 20^{\prime} \mathrm{N}, 130^{\circ} 12^{\prime} \mathrm{E}, 15$ Oct $2001 ; 6$ solitary zooids and 38 aggregate zooids, East Sea, $37^{\circ} 00^{\prime} \mathrm{N}, 129^{\circ} 30^{\prime} \mathrm{E}$, 23 Sep 2002; 1 solitary zooid, Korea strait, $33^{\circ} 59^{\prime} \mathrm{E}, 128^{\circ}$ $17^{\prime} \mathrm{N}, 24$ Sep 2002; 65 solitary zooids and 2 aggregate zooids, East China Sea, $29^{\circ} 15^{\prime} \mathrm{N}, 125^{\circ} 50^{\prime} \mathrm{E}, 24$ Sep 2002; 13 solitary zooids, South Korea, $34^{\circ} 57^{\prime} \mathrm{N}, 129^{\circ} 22^{\prime} \mathrm{E}, 3$ Dec 2002; 1 aggregate zooid, South Korea, $34^{\circ} 05^{\prime} \mathrm{N}, 127^{\circ} 96^{\prime} \mathrm{E}$, 1 Sep 2008; 1 aggregate zooid, East Sea, $35^{\circ} 46^{\prime} 21.54^{\prime \prime} \mathrm{N}, 129^{\circ} 31^{\prime}$ $39.69^{\prime \prime}$ E, 22 Oct 2010.

Description. Solitary zooid: $3-14 \mathrm{~mm}$ long, excluding posterior projections (Fig. 3A, B). Body oval or cylindrical (Fig. 3A, B). Test smooth (Fig. 3A, B). Atrial palps bifurcate (Fig. $3 C)$. Posterior projections very long that almost same length with trunk (Fig. 3A, B). Medioventral projections well developed and lower one longer than upper one (Fig. 3B). Lateral projections not exist. All projections echinate (Fig. 3A-C). Body muscles thin (Fig. 3A, B).

Aggregate zooid (Fig. 3D): 1-13 mm long. Body oval with projected posteriorly. Test smooth. Attachment organs variable in number and protruding beyond test, especially right anterior and left posterior. Nucleus oval.

Distribution. Pacific Ocean, Atlantic Ocean, and Indian Ocean.

\section{1*Thaila rhomboides (Quoy and Gaimard, 1824) (Fig. 4A-D)}

Salpa rhomboides Quoy and Gaimard, 1824: 479 (cited from van Soest, 1998; Kott, 2005).

Thalia rhomboides: Chihara and Murano, 1997: 1365; van Soest, 1998: 238, fig. 14.3, table 14.3.

Materials examined. 17 solitary zooids and 14 aggregate zooids, Korea strait, $32^{\circ} 29^{\prime} \mathrm{N}, 127^{\circ} 29^{\prime} \mathrm{E}, 8$ May 2001 ; 1 solitary zooid, South Korea, $35^{\circ} 24^{\prime} \mathrm{N}, 129^{\circ} 34^{\prime} \mathrm{E}, 3$ Dec 2001; 40 solitary zooids and 7 aggregate zooids, Korea strait, $32^{\circ}$ $29^{\prime} \mathrm{N}, 127^{\circ} 29^{\prime} \mathrm{E}, 24$ Sep 2002; 1 aggregate zooid, Korea strait, $33^{\circ} 59^{\prime} \mathrm{N}, 128^{\circ} 17^{\prime} \mathrm{E}, 24$ Sep 2002 ; 46 solitary zooids and 12 aggregate zooids, Korea strait, $31^{\circ} 59^{\prime} \mathrm{N}, 127^{\circ} 29^{\prime} \mathrm{E}$, 2 Dec 2002; 1 aggregate zooid, South Korea, $34^{\circ} 05^{\prime} \mathrm{N}, 127^{\circ}$ 96'E, 1 Sep 2008; 2 solitary zooids, South Korea, $35^{\circ} 20^{\prime} \mathrm{N}$, $130^{\circ} 12^{\prime} \mathrm{E}, 15$ Oct $2001 ; 1$ solitary zooid and 1 aggregate zooid, East Sea, $38^{\circ} 30^{\prime} \mathrm{N}, 129^{\circ} 49^{\prime} \mathrm{E}, 15$ Oct $2001 ; 1$ solitary zooid and 1 aggregate zooid, Yeosu, $34^{\circ} 42^{\prime} \mathrm{N}, 127^{\circ} 40^{\prime} \mathrm{E}$, Nov 2002; 1 aggregate zooid, $35^{\circ} 47^{\prime} 33.06^{\prime \prime} \mathrm{N}, 129^{\circ} 32^{\prime} 27.37^{\prime \prime} \mathrm{E}$, 22 Oct 2010.

Description. Solitary zooid: $3-12 \mathrm{~mm}$ long, excluding posterior projections (Fig. 4A, B). Body elongated cylindrical (Fig. 4A, B). All test echinate, especially projections (Fig. 4A-C). Atrial palps bifurcate (Fig. 4C). Medioventral projec- tions well developed and lower one longer than upper one (Fig. 4A, B). Lateral projections well developed (Fig. 4A, B). Body muscles thick (Fig. 4A, B).

Aggregate zooid (Fig. 4D): 1-2 mm long. Body pentagonal shape with sharp-edged with Echinated. Sharply pointed posterior. Test relatively hard. Attachment argans not protruding test well. Nucleus oval.

Distribution. Pacific Ocean, Atlantic Ocean, and Indian Ocean.

${ }^{2 *}$ Thaila sibogae van Soest, 1973 (Fig. 5A-C)

Thalia sibogae van Soest, 1973: 204; van Soest, 1998: 238, fig. 14.3, table 14.3.

Materials examined. 9 solitary zooids, South Korea, $35^{\circ}$ $20^{\prime} \mathrm{N}, 130^{\circ} 12^{\prime} \mathrm{E}, 8$ Sep 2002.

Description. Solitary zooid: $7-11 \mathrm{~mm}$ long, excluding posterior projections (Fig. 5A, B). Body oval or cylindrical (Fig. 5A, B). All test echinate, especially projections (Fig. 5A, B). Atrial palps bifurcate (Fig. 5C). Medioventral projections short, lower one longer than upper one (Fig. 5B). Lateral projections very short or not developed. Posterior projections relatively short (Fig. 5A, B). Body muscles very thick (Fig. 5A, B).

Distribution. Pacific Ocean and Indian Ocean.

Remarks. Aggregate zooids of this species were not collected during this study.

\section{Key to the life cycles of Thalia species}

1. Stolon presence at ventral or posterior, egg and embryo absent, attachment organ absent …………... solitary zooid

- Stolon absent, eggs or embryo presence at right, attachment organ presence …………............................ aggregate zooid

Solitary zooid

Key to the species of Thalia in Korea

1. Atrial palps simple and straight ………..... T. democratica

- Atrial palps bifurcate ……………………………………. 2

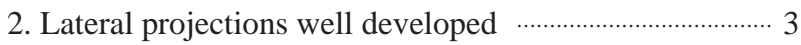

- Lateral projections not exist or not developed ……......... 4

3. Test smooth, muscle bands thin …………............ T. cicar

- All test echinate, muscle bands thick …… T. rhomboides

4. Test smooth, muscle bands thin, MIV and MV fused in mid-dorsal line ......................................... T. orientalis

- All test echinate, muscle bands thick, MIV and MV touching in mid-dorsal line ……................................ T. sibogae

Aggregate zooid

1. Pentagonal shaped body ……..................... T. rhomboides

Korean name: ${ }^{1 *}$ 네모탈리아살파 (신칭), ${ }^{2 *}$ 넓은띠탈리아살파 (신칭) 

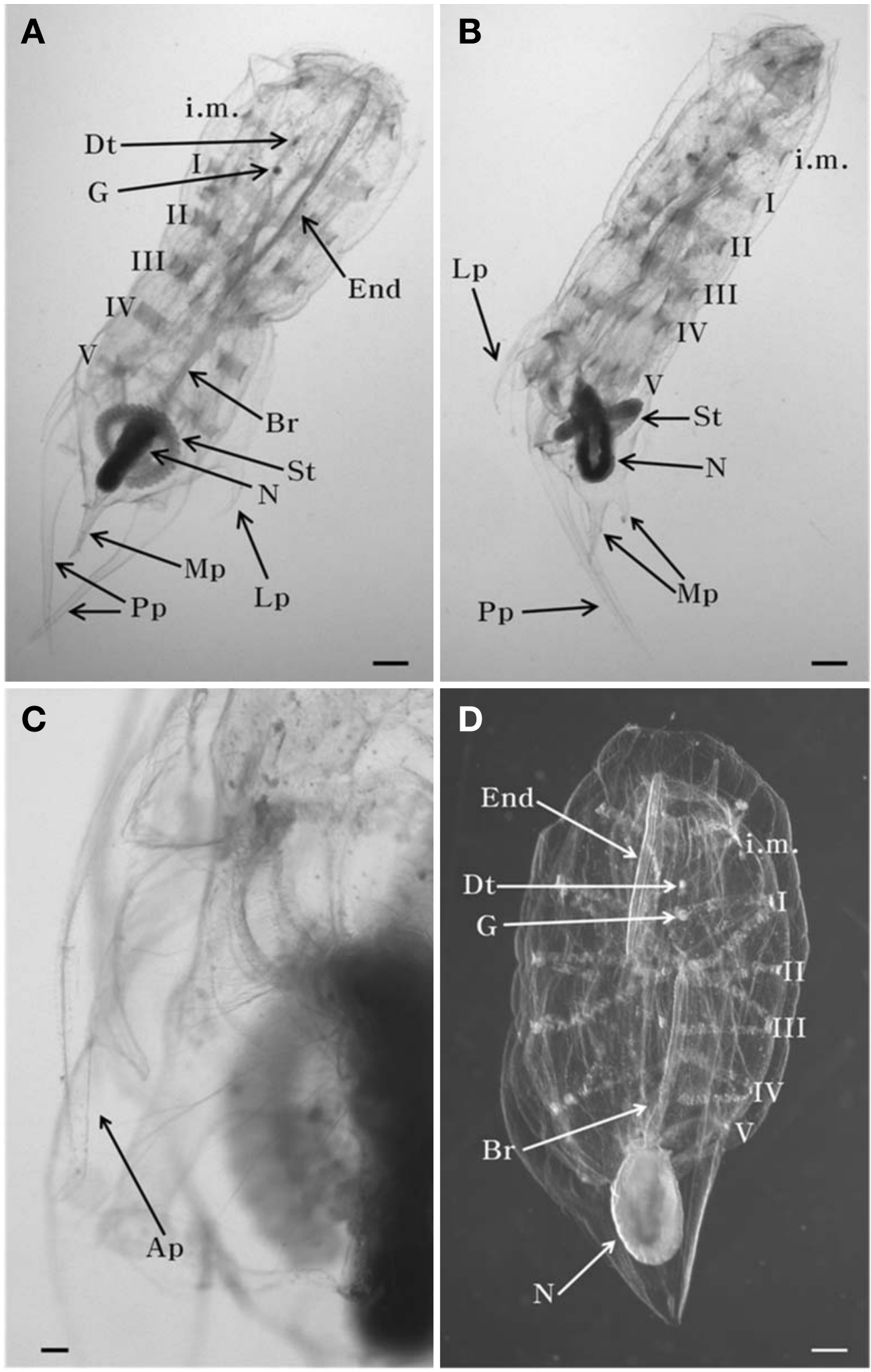

Fig. 4. Thalia rhomboides. A-C, Solitary zooid. A, Ventral view; B, Lateral view; C, Lateral part. D, Aggregate zooid. ventral view. $\mathrm{Ap}$, atrial projection; $\mathrm{Br}$, branchial septum; $\mathrm{Dt}$, dorsal turbercle; End, endostyle; G, ganglion; i.m., intermediate muscle; Lp, lateral projection; Mp, medioventral projection; $\mathrm{N}$, nucleus; Pp, posterior projection; St, stolon; I- $\mathrm{V}$, body muscle. Scale bars: $\mathrm{A}$, $\mathrm{B}=0.5$ $\mathrm{mm}, \mathrm{C}=0.1 \mathrm{~mm}, \mathrm{D}=0.2 \mathrm{~mm}$. 

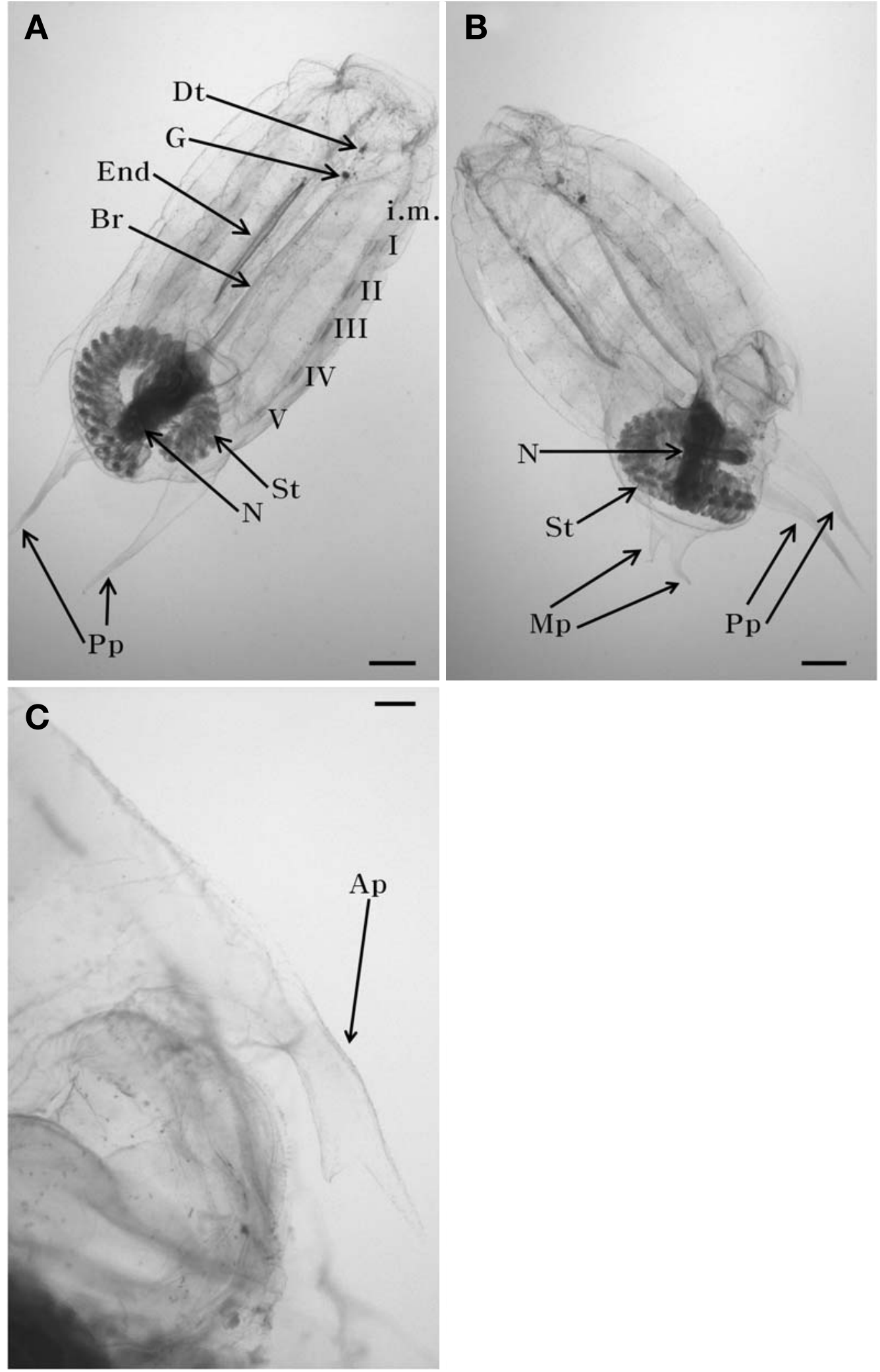

Fig. 5. Thalia sibogae. A-C, Solitary zooid. A, Ventral view; B, Lateral view; C, Lateral part. Ap, atrial projection; $B r$, branchial septum; Dt, dorsal turbercle; End, endostyle; G, ganglion; i.m., intermediate muscle; Mp, medioventral projection; $\mathrm{N}$, nucleus; Pp, posterior projection; St, stolon I-V, body muscle. Scale bars: $A, B=1 \mathrm{~mm}, C=0.2 \mathrm{~mm}$. 
- Oval body with a projection at posterior end or not …... 2

2. Nucleus bearing a projection at posterior end (nucleus projection) T. democratica

- Nucleus oval without nucleus projection ……….............. 3

3. Body with round posterior end ………….............. T. cicar

- Body with projected posterior end ………....... T. orientalis

\section{REFERENCES}

Berrill NJ, 1950. The Tunicata, with an account of the British species. Ray Society, London, pp. 1-354.

Chihara M, Murano M, 1997. An illustrated guide to marine plankton in Japan. Tokai University Press, Tokyo, pp. 11574.

Esnal GB, Daponte MC, 1999. Salpida. In: South Atlantic zooplankton (Ed., Boltovskoy D). Backhuys Publishers, Leiden, pp. 1409-1421.

Fraser JH, 1947. Thaliacea, I. Family: Salpidae. Zooplankton Sheet, CIEM, 9:1-4.

Gosner KL, 1971. Guide to identification of marine and estuarine invertebrates: Cape Hatteras to the Bay of Fundy. John Wiley and Sons, Inc., New York, pp. 1-693.

Kim S, Won JH, Kim CB, 2010a. A new record of Iasis zonaria (Salpida: Salpidae) in Korean waters. Korean Journal of Systematic Zoology, 26:161-163.

Kim S, Won JH, Lee J, Kim CB, 2010b. A new record of Ihlea punctata and redescription of Salpa fusiformis (Salpida: Salpidae) in Korean waters. Korean Journal of Systematic Zoology, 26:353-356.

Kott P, 2005. Catalogue of tunicata in Australian waters. Australian Biological Resources Study, Department of the Environment and Heritage, Canberra, pp. 1-301.

Madin LP, 1991. Distribution and taxonomy of zooplankton in the Alboran Sea and adjacent western Mediterranean. A literature survey and field guide. Report for Harbor Branch Oceanographic Institution and Naval Oceanographic and Atmospheric Research Laboratory. Technical Report 91-26. Wood Hole Oceanographic Institution, Woods Hole, MA, pp. 1-148.

Rho BJ, 1967. Taxonomic studies on the Tunicata. Korea Culture Research Institute Publication, 10:361-371.

The Korean Society of Systematic Zoology, 1997. List of animals in Korea (excluding insects). Academy Publishing Co., Seoul, pp. 1-489.

Thompson H, 1948. Pelagic tunicates of Australia. Commonwealth Council for Scientific and Industrial Research, Melbourne, pp. 1-196.

Tokioka T, 1937. Notes on salpas and doliolums occurring on the Pacific coast of middle Japan. Annotationes Zoologicae Japonenses, 16:219-235.

Tokioka T, 1967. Pacific Tunicata of the United States National Museum. United States National Museum Bulletin, 251:1247.

van Soest RWM, 1973. A new species in the genus Salpa Forskal, 1775 (Tunicata, Thliacea). Beaufortia, 21:9-15.

van Soest RWM, 1998. The cladistic biogeography of salps and pyrosomas. In: The biology of pelagic tunicates (Ed., Bone Q). Oxford University Press, Oxford, pp. 232-249.

Yamaji I, 1984. Illustrations of the marine plankton of Japan. Hoikusha Publishing Co., Osaka, pp. 1-537.

Yount JL, 1954. The taxonomy of the Salpidae (Tunicata) of the central Pacific Ocean. Pacific Science, 8:276-330.

Zheng Z, 1989. Marine planktology. China Ocean Press, Beijing, pp. 1-454. 